\title{
Formation of and countermeasures for subacute coronary stent thrombosis in elderly diabetic patients
}

\author{
Z.-F. Li ${ }^{1}$, Y.-P. Zhang ${ }^{2}$, Z.-Q. Qin², X.-L. Li ${ }^{1}$, C.-H. Gao ${ }^{1}$, S. Yang ${ }^{1}$ and \\ Z.-J. Chen ${ }^{1}$
}

${ }^{1}$ Department of Cardiology, Yongchuang Hospital, Chongqing Medical University, Chongqing, China

${ }^{2}$ Department of Cardiology, People's Hospital of Tongliang County, Chongqing, China

Corresponding author: Y.-P. Zhang

E-mail: 1zf56888@163.com

Genet. Mol. Res. 13 (3): 6107-6112 (2014)

Received July 30, 2013

Accepted November 1, 2013

Published August 7, 2014

DOI http://dx.doi.org/10.4238/2014.August.7.26

\begin{abstract}
The aim of this study was to evaluate and investigate the pathogenetic mechanism and countermeasures of subacute thrombosis (SAT) after coronary stenting in elderly diabetic patients. The clinical characteristics and pathogenetic mechanisms in 3 cases of SAT after stent implantations in elderly diabetic patients were retrospectively examined to determine the treatment strategies for SAT. Among 98 patients with diabetes who had coronary stents implanted or were $>60$ years of age, three $(3.06 \%)$ had SAT. One case of SAT was diagnosed by angiography; coronary balloon dilatation, thrombolysis, and reperfusion resulted in full recovery in this case. The second case involved potential SAT, and in the third case, SAT was not ruled out. Two cases were characteristic of ST-segment elevation myocardial infarction, and one case, in which SAT was not ruled out, resulted in sudden death. SAT within a stent may be related to intraoperative stent
\end{abstract}


malapposition caused by a grade $\mathrm{C}$ lesion, age, diabetes, chronic total occlusion, or postoperative irregular administration of medication.

Key words: Elderly diabetes; Coronary stent; Thrombosis

\section{INTRODUCTION}

Stent thrombosis (ST) is serious complication of coronary intervention treatment that has an incidence of only $0.9-1 \%$ but a poor prognosis (Trabattoni et al., 2007). The higher incidence of subacute thrombosis (SAT) with increase in age, hypertension, and diabetes could lead to the deaths of one-third to one-half of the affected patients. According to stent implantation time, ST was divided into acute $(<24 \mathrm{~h})$, subacute (1-30 days), end stage (1-12 months), and very end stage ( $>1$ year) thrombosis (Moussa et al., 1997; Farb et al., 2003). The incidence of SAT in patients with ST is approximately 50\% (Trabattoni et al., 2007). SAT after percutaneous coronary intervention (PCI) occurs mainly as follows: 1) Independent factors for SAT include diabetes, renal insufficiency, acute coronary syndrome, ST-segment elevation myocardial infarction, long lesions, and left anterior descending branch (de la Torre-Hernández et al., 2008). 2) Stent factors: incomplete stent expansion, incomplete stent coverage of a lesion, residual stenosis, and residual dissection after stenting all cause thrombosis. Metal stents promote thrombosis formation. Drug-coated stents may inhibit endometrial hyperplasia and reduce the restenosis rate; however, endothelialization may be delayed. The compatibility and endothelial incomplete repair of the stents may also induce blood hypercoagulability. These factors could all lead to an increased risk of thrombosis (Harper, 2007). 3) Anticoagulant therapy: anticoagulant drug discontinuation or resistance to clopidogrel hydrogen and aspirin (Nguyen et al., 2005). 4) Intraoperative operating factors: guidance of a steel wire through intraoperative PCI, balloon dilatation, and stent release could all damage blood vessels, leading to endometrial tearing, which could cause exposure to subendometrial collagenous fibers, activate the coagulation system, and then lead to thrombus formation (Neumann et al., 1996). The diameter of the target blood vessel and the degree of residual stenosis are also important factors affecting stent thrombosis. Some studies found that incomplete stent expansion was an independent risk factor of postoperative stent thrombosis (Cheneau et al., 2003). Ischemia reperfusion injury mechanisms might also be involved in stent thrombosis.

The purpose of this study was to evaluate and investigate the pathogenetic mechanism of and countermeasures for subacute thrombosis in elderly patients with diabetes combined with coronary diseases. Advanced age, diabetes, chronic total occlusion (CTO), diffuse calcification, bifurcation lesions, and anticoagulant treatment discontinuation were pathogenetic mechanisms of subacute thrombosis, providing references for the preventive treatment of subacute stent thrombosis after PCI.

The clinical characteristics and treatment of three patients with SAT after undergoing stenting from October 2010 to August 2011 in our department were reported as follows.

\section{MATERIAL AND METHODS}

\section{General data}

From October 2010 to August 2011, 98 patients aged $>60$ years (with or without dia- 
betes) underwent PCI in our department, three of whom (two men and one woman) had SAT in the coronary stent. These patients were aged 61-82 years (mean age, 73.3 years), as shown in Table 1.

\begin{tabular}{lccc}
\multicolumn{2}{l}{ Table 1. General clinical data of three patients with coronary stent subacute thrombosis. } & Case 3 \\
\hline Items & Case 1 & Case 2 & 82 \\
\hline Age (years) & 61 & 78 & No \\
Hypertension & Yes & Yes & Yes \\
Diabetes & Yes & Yes & Yes \\
CTO & Yes & No & Old MI \\
Diagnosis & Old MI & 42 & 50 \\
Left ventricular ejection fraction & 54 & S
\end{tabular}

$\mathrm{CTO}=$ chronic total occlusion; $\mathrm{MI}=$ myocardial infarction; $\mathrm{UAP}=$ unstable angina pectoris.

\section{PCI treatment}

The three patients underwent elective operation and were administered preoperative $100 \mathrm{mg} /$ day aspirin and $75 \mathrm{mg}$ /day clopidogrel for more than 1 month. All of the patients had multivessel disease that had nine target lesion parts and was classified as grade $\mathrm{C}$ lesions according to the American College of Cardiology and American Heart Association. Two patients had CTO: nine drug-eluting stents (DES) were implanted, without postoperative residual stenosis, with forward flow to thrombolysis in myocardial infarction (TIMI) grade 3 . Three patients underwent full pre-dilatation before stenting, and two patients underwent post-dilatation after stenting. Because of the tortuous and calcified lesions, a high-pressure balloon of the same size as the stent was used. Postoperative stent inflation was good, without residual stenosis, as shown in Table 2.

\begin{tabular}{lccc}
\multicolumn{4}{c}{ Table 2. Coronary artery lesions and PCI treatment materials of three patients with coronary stent SAT. } \\
\hline Items & Case 1 & Case 2 & Case 3 \\
\hline PCI pathogenesis (days) & 70 & 30 & 84 \\
Pathological vessels & 3 & 2 & 3 \\
Stent-implanted parts & LAD middle and distal part, & LAD middle part, & LM middle part, \\
& LCX proximal part, & RCA middle part & LAD middle and distal part, \\
Lesion characteristics & RCA middle part & Calcified tortuous & LCX middle part \\
Longest lesions (mm) & Calcified diffuse CTO & 18 & Calcified diffuse CTO \\
SAT vessel & 38 & LAD & 20 \\
SAT stent diameter (mm) & LAD & 3.0 & LCX \\
SAT stent length (mm) & 2.75 & 24 & 2.5 \\
Post-dilation & 24 & Yes & 18 \\
Malapposition & Yes & No & Yes \\
Preoperative TIMI flow & No & 3 & No \\
Postoperative TIMI flow & 0 & 3 & 0 \\
\hline
\end{tabular}

PCI = percutaneous coronary intervention; SAT = subacute thrombosis; TIMI = thrombolysis in myocardial infarction; LAD = left anterior descending artery; LCX = left circumflex artery; RCA = right coronary artery; $\mathrm{CTO}=$ chronic total occlusion; LM = left main coronary artery.

\section{RESULTS}

Postoperative symptoms occurred in three patients for 2-10 days. In case 1, continu- 
ous $3.0 \times 21$ and $2.75 \times 24 \mathrm{~mm}$ DES in the middle and distal part of the left anterior descending (LAD) artery, $3.0 \times 18 \mathrm{~mm}$ DES in the left circumflex (LCX) artery, and $3.0 \times 15 \mathrm{~mm}$ DES in the right coronary artery were implanted. On postoperative day 6 , there was no angina, cardiac function was class I, and the clinical manifestation was very stable. On postoperative day 7, the patient experienced sudden death after a cold. In case 2, 3.0 x 24 and $3.0 \times 28 \mathrm{~mm}$ DES were implanted in the LAD and LCX. Postoperative angina was terminated. At 6 days postoperatively, the patient self-discontinued the aspirin and clopidogrel. On postoperative day 10 , the patient experienced myocardial infarction; after $10 \mathrm{~h}$, he was diagnosed with acute myocardial infarction in our department. Anterior descending stent thrombosis was observed on coronary angiography, with forward flow to TIMI grade 0 . Immediate and repeated stent percutaneous transluminal coronary angioplasty (PTCA) was performed, and 200,000 U coronary urokinase was injected. Residual stenosis was not seen on coronary angiography, and the forward flow was recovered to TIMI grade 3.

In case 3, a $3.0 \times 24 \mathrm{~mm}$ DES was implanted in the LAD, a $3.5 \times 15 \mathrm{~mm}$ DES was implanted in the left main, and a $2.5 \times 18 \mathrm{~mm}$ DES was implanted in the LCX artery. On postoperative day 5 , the patient experienced chest pain for $>20 \mathrm{~min}$; II, III, aVF, and STsegment elevation was $>0.1 \mathrm{~mm}$; and CK-MB was increased significantly to more than twice the normal value. The patient was diagnosed with acute inferior diaphragmatic infarction. Because the chest pain was relieved after approximately $30 \mathrm{~min}$ without abnormal vital signs and because the patient was very elderly and his family members refused coronary angiography, PTCA, and thrombolysis, conservative treatment was performed. After 2 weeks, the patient's condition improved and he was discharged. ST treatments and results are shown in Table 3.

Table 3. Treatments and results of 3 patients with coronary stent SAT.

\begin{tabular}{|c|c|c|c|}
\hline Items & Case 1 & Case 2 & Case 3 \\
\hline SAT occurrence time (days) & 7 & 10 & 3 \\
\hline SAT symptom & Sudden death & AMI & AMI \\
\hline Coronary angiogram & No & Yes & No \\
\hline PCI treatment & No & Yes & No \\
\hline Clinical results & Sudden death & Improved & Improved \\
\hline
\end{tabular}

$\mathrm{SAT}=$ subacute thrombosis; $\mathrm{PCI}=$ percutaneous coronary intervention; $\mathrm{AMI}=$ acute myocardial infarction.

\section{DISCUSSION}

ST is a serious complication of coronary intervention treatment and often endangers patients' lives. The mechanism underlying ST involves multiple factors, so prevention is not very effective. As reported in the literature (Moussa et al., 1999; Cheneau et al., 2003; Buonamici et al., 2007; Machecourt et al., 2007), ST formation is related to many factors such as acute coronary syndrome, plaque rupture, platelet activation, and increased agglutinant release in vivo. In some cases, aspirin and clopidogrel were not fully used and were even discontinued (case 2 in this paper). Other factors are agedness, diabetes, and chronic renal insufficiency. The DES effectively reduces the rate of stent restenosis and major adverse cardiac events of the target lesion (or target vessel) revascularization by inhibiting endometrial hyperplasia. However, the ST incidence of DES, especially SAT, remains a concern. The ESTROFA registry in Spain (de la Torre-Hernández et al., 2008) showed that the incidence of ST is $1.2 \%$ and that $50 \%$ of these cases involved SAT. DES was used in three cases of SAT in the present study. 
The Academic Research Consortium (ARC) (Cutlip et al., 2007), consisting of data from the United States and Europe in 2007, divided ST into the following three categories. The first is definite or confirmed ST: acute coronary syndrome, definite radiographic findings of ST, or thrombosis in a stent within $5 \mathrm{~mm}$ of the proximal or distal portion confirmed by autopsy. The second is probable ST: any unexplained death within 30 days; postoperative myocardial infarction related to definite myocardial ischemia in the coronary region supplied by the implanted stent, without other obvious reasons and without imaging findings to confirm stent thrombosis. The third is possible ST: any unexplained death within 30 days. Case 2 in this paper involved definite SAT with a definite reason for discontinuation of clopidogrel, aspirin, or some other antiplatelet agent. The ARC believes that SAT had a direct relationship with stent malapposition, residual dissection, or failure of a stent to completely cover a lesion. Cases 1 and 3 in this article involved occlusive diseases, making it difficult to avoid residual dissection. Extensively calcified and tortuous vessels caused by advanced age and diabetes, and the small target vessels were all factors for stent malapposition. Although all such lesions were treated with full pre- and post-dilatation, it was difficult to avoid individual stent malapposition. Compared with patients who have coronary disease and normal sugar metabolism, these types of patients are susceptible to SAT because of a large number of lesions and implanted stents (Schühlen et al., 1998; Buonamici et al., 2007). Because of diffuse lesions in the vessels of patients with diabetes, the pre-expanded balloon had a smaller maximum diameter and the implanted stent had a longer length and smaller diameter during PCI (Goraya et al., 2002). These might be causes of the high SAT incidence in patients with advanced age or diabetes.

Analysis of the above three cases of SAT highlighted the following results: 1) antiplatelet therapy should be strictly regulated;2) in high-risk elderly patients who have diabetes, CTO, or diffusely calcified or bifurcated lesions, PCI or coronary artery bypass grafting should be performed after assessing SYNTAX score and radiography results (Sianos et al., 2005; Ong et al., 2006); and 3) immediate and decisive PCI treatment should be provided for patients with SAT.

\section{Conflicts of interest}

The authors declare no conflict of interest.

\section{REFERENCES}

Buonamici P, Marcucci R, Migliorini A, Gensini GF, et al. (2007). Impact of platelet reactivity after clopidogrel administration on drug-eluting stent thrombosis. J. Am. Coll. Cardiol. 49: 2312-2317.

Cheneau E, Leborgne L, Mintz GS, Kotani J, et al. (2003). Predictors of subacute stent thrombosis: results of a systematic intravascular ultrasound study. Circulation 108: 43-47.

Cutlip DE, Windecker S, Mehran R, Boam A, et al. (2007). Clinical end points in coronary stent trials: a case for standardized definitions. Circulation 115: 2344-2351.

de la Torre-Hernández JM, Alfonso F, Hernandez F, Elizaga J, et al. (2008). Drug-eluting stent thrombosis: results from the multicenter Spanish registry ESTROFA (Estudio Espanol sobre Trombosis de Stents Farmacoactivos). J. Am. Coll. Cardiol. 51: 986-990.

Farb A, Burke AP, Kolodgie FD and Virmani R (2003). Pathological mechanisms of fatal late coronary stent thrombosis in humans. Circulation 108: 1701-1706.

Goraya TY, Leibson CL, Palumbo PJ, Weston SA, et al. (2002). Coronary atherosclerosis in diabetes mellitus: a population- 
based autopsy study. J. Am. Coll. Cardiol. 40: 946-953.

Harper RW (2007). Drug-eluting coronary stents - a note of caution. Med. J. Aust. 186: 253-255.

Machecourt J, Danchin N, Lablanche JM, Fauvel JM, et al. (2007). Risk factors for stent thrombosis after implantation of sirolimus-eluting stents in diabetic and nondiabetic patients: the EVASTENT Matched-Cohort Registry. J. Am. Coll. Cardiol. 50: 501-508.

Moussa I, di Mario C, Reimers B, Akiyama T, et al. (1997). Subacute stent thrombosis in the era of intravascular ultrasound-guided coronary stenting without anticoagulation: frequency, predictors and clinical outcome. J. Am. Coll. Cardiol. 29: 6-12.

Moussa I, Oetgen M, Roubin G, Colombo A, et al. (1999). Effectiveness of clopidogrel and aspirin versus ticlopidine and aspirin in preventing stent thrombosis after coronary stent implantation. Circulation 99: 2364-2366.

Neumann FJ, Ott I, Gawaz M, Puchner G, et al. (1996). Neutrophil and platelet activation at balloon-injured coronary artery plaque in patients undergoing angioplasty. J. Am. Coll. Cardiol. 27: 819-824.

Nguyen TA, Diodati JG and Pharand C (2005). Resistance to clopidogrel: a review of the evidence. J. Am. Coll. Cardiol. 45: 1157-1164.

Ong AT, Serruys PW, Mohr FW, Morice MC, et al. (2006). The SYNergy between percutaneous coronary intervention with TAXus and cardiac surgery (SYNTAX) study: design, rationale, and run-in phase. Am. Heart J. 151: 1194-1204.

Schühlen H, Kastrati A, Dirschinger J, Hausleiter J, et al. (1998). Intracoronary stenting and risk for major adverse cardiac events during the first month. Circulation 98: 104-111.

Sianos G, Morel MA, Kappetein AP, Morice MC, et al. (2005). The SYNTAX Score: an angiographic tool grading the complexity of coronary artery disease. EuroIntervention 1: 219-227.

Trabattoni D, Fabbiocchi F, Montorsi P, Ravagnani P, et al. (2007). Stent thrombosis after sirolimus- and paclitaxeleluting stent implantation in daily clinical practice: analysis of a single center registry. Catheter. Cardiovasc. Interv. 70: 415-421. 